\title{
Giant Schwannoma of the Median Nerve: A Case Report and Literature Review
}

\author{
Jennyfer Paulla Galdino Chaves ${ }^{1,0}$ Luis Henrique Albuquerque Sousa ${ }^{1}$ \\ Emanuella Roberta Iná Cirino ${ }^{1,2}$ Robinson Antonio Menegotto Marques ${ }^{1}$
}

${ }^{1}$ Neurosurgery Department, Cajuru University Hospital, Curitiba, Brazil

2State University of West Paraná (UNIOESTE), Cascavel, Brazil

\begin{abstract}
Address for correspondence Jennyfer Paulla Galdino Chaves, MD, Neurosurgery Department, Cajuru University Hospital, 300, São José-Cristo Rei, Curitiba, PR 80050-350, Brazil (e-mail: jennyfergaldino@hotmail.com).
\end{abstract}

\begin{abstract}
Keywords

- schwannoma

- nerve sheath tumor

- surgery

Peripheral nerve sheath tumors (PNST) develop sporadically or are associated with genetic disorders and affect individuals mainly between the third and sixth decades of life. PNST affect one or multiple fascicles. The benign ones have slow and painless growth, preceding in years the diagnosis. Diagnosis is often confused with other soft tissue tumors, such as fibroids and lipomas. We present a rare case of giant schwannoma of the median nerve. The patient had a progressive local growth with minimal neurological impairment.
\end{abstract}

\section{Introduction}

Peripheral nerve sheath tumors (PNST) develop sporadically or are associated with genetic disorders ${ }^{1,2}$ and affect individuals mainly between the third and the sixth decades of life. ${ }^{3}$ The most common are benign tumors, accounting for $10 \%$ of benign soft tissue tumors. Schwannomas are the main representatives and originate from the deficiency of merlin tumor suppressor protein in Schwann cells due to loss of heterozygosity in the NF2 gene. ${ }^{3}$ Next, we have the neurofibromas that arise from the mutation or loss of heterozygosity of the NF1 gene, resulting in upregulation of the RAS protein. ${ }^{1,3}$

Among the histological variants of benign tumors, there is a plexiform neurofibroma that represents a higher risk of malignant evolution, especially in patients with neurofibromatosis. ${ }^{1}$ Only $10 \%$ of these subjects develop the neoplasia; however, half of malignant peripheral nerve sheath tumors (MPNST) contain the NF1 mutation..$^{1,3}$ This relation presents a worse prognosis for MPNST, neoplasms with great metastatic potential, and for local recurrence. ${ }^{1}$

Peripheral nerve sheath tumors affect one or multiple fascicles. The benign ones have slow and painless growth, preceding in years the diagnosis. ${ }^{1}$ They may, in more advanced cases or depending on the affected area, manifest with pain, paresthesia, and weakness. And when there is motor deficit, pain at rest, and accelerated growth, malignancy should be suspected. ${ }^{1,3}$
Diagnosis is often confused with other soft tissue tumors, such as fibroids and lipomas. ${ }^{1}$ Therefore, the history and clinical examination associated with complementary exams such as electrophysiological study and imaging are fundamental. ${ }^{2}$ Magnetic resonance imaging (MRI) is the examination of choice during the investigation as it determines the extent and relationship of the tumor to the adjacent structures. Percutaneous biopsy allows tumor characterization, but it offers risks such as neurological injury and translocation of tumor cells and is not recommended. ${ }^{2,3}$

Expectant management is possible for benign lesions; however, there is a risk of malignant progression, evolution with neurological deficit, and aesthetic discomfort. ${ }^{3}$ Therefore, the most used and curative treatment is surgical resection.

\section{Case Report}

A 42-year-old man presented with progressive protrusion during 20 years in the wrist region. He referred only local pain. In neurologic exam, he had a strength grade IV for thumb opposition and finger flexion ( - Fig. 1). He underwent an MRI that showed a lesion suggestive of peripheral nerve sheath tumors in the median nerve ( - Fig. 2). He was subjected to a tumoral resection ( - Figs. 3 and $\mathbf{4}$ ). It was possible to leave the fascicles intact during the dissection. We used intraoperative ultrasound ( - Fig. 5) where we could see the published online September 16, 2019
DOI https://doi.org/ 10.1055/s-0039-1696591 ISSN 2454-6798.
C2019 Spring Hope Cancer Foundation \& Young Oncologist Group of Asia

\section{License terms}

()(1) $\Theta \circledast$ 


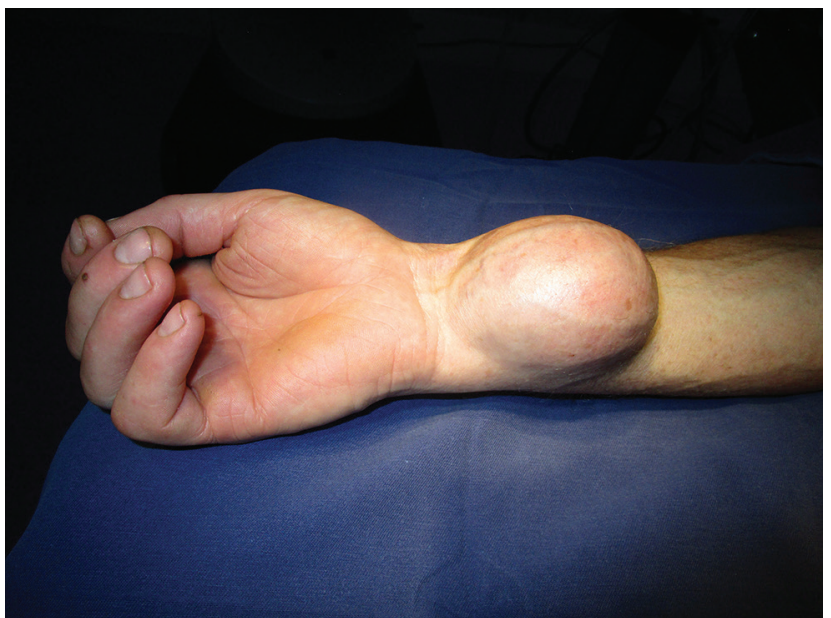

Fig. 1 Macroscopic lesion visualization.

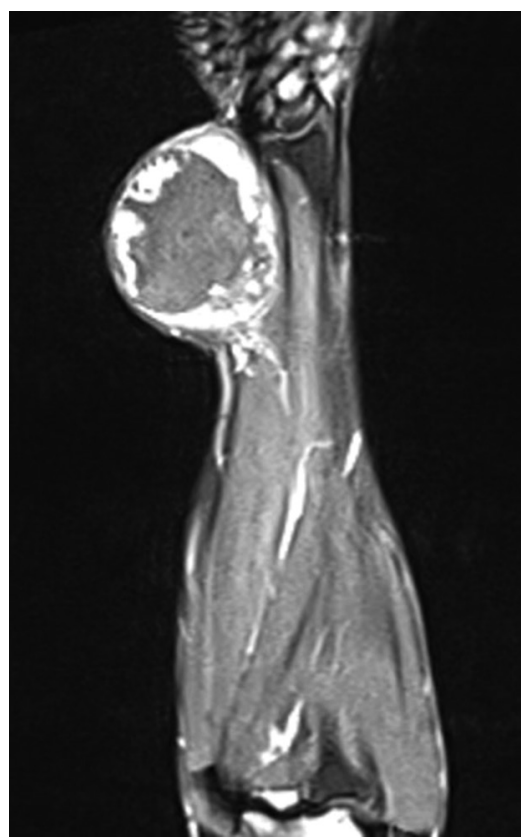

Fig. 2 Magnetic resonance imaging showing a lesion suggestive of peripheral nerve sheath tumors in the median nerve.

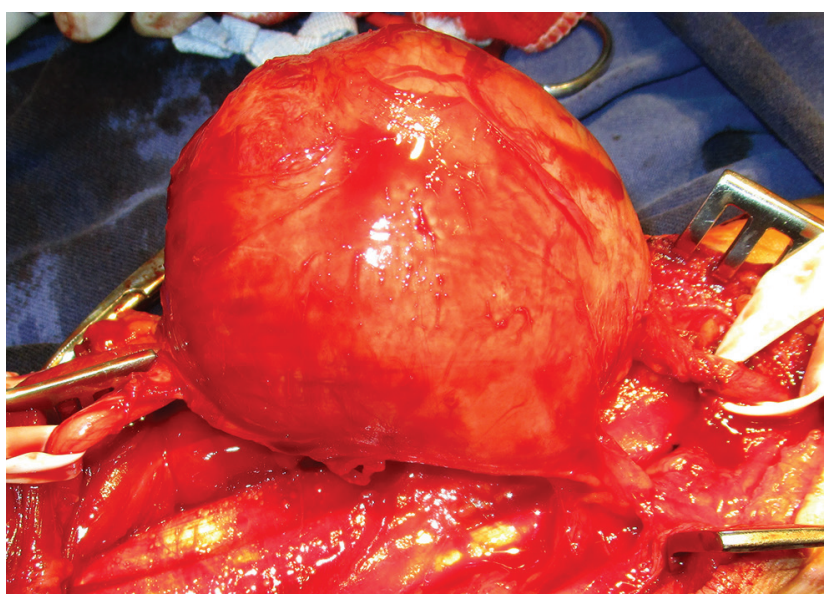

Fig. 3 Tumor resection.

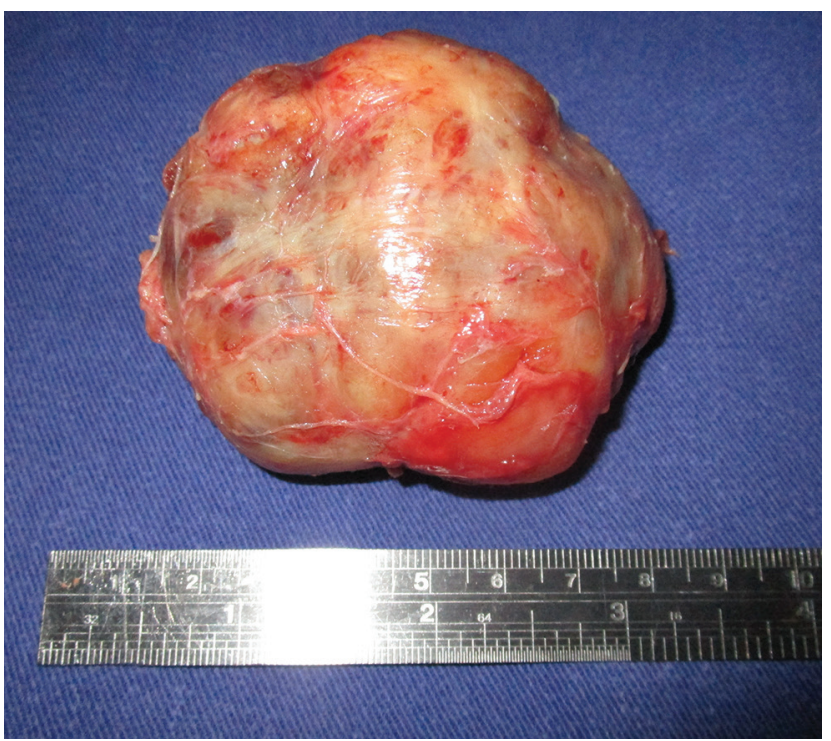

Fig. 4 Tumor resection.

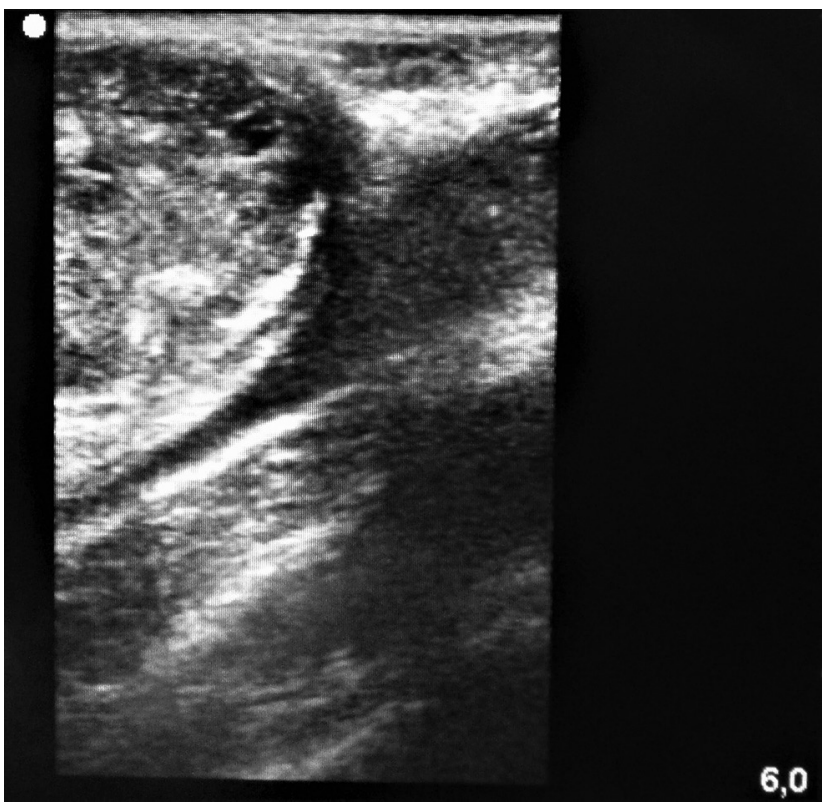

Fig. 5 Intraoperative ultrasound.

lesion attached to the median nerve and get better visualization when looking for tumor residues. The nerve graft was not necessary. A zetoplasty was utilized for the reduction of redundant skin after tumor resection. The patient did not report new neurological impairment. The anatomopathology was compatible with Schwannoma.

\section{Discussion}

Schwannomas, formerly called neurilemomas, also manifest themselves between the genders, especially between 20 and 70 years. ${ }^{4}$ They occur usually in the head and neck region and more rarely in the upper limbs, corresponding to 5 to $8 \%$ of all tumors of the upper extremities. ${ }^{4,5}$ The flexor region has 
abundant nerve structures so it is more affected, as demonstrated in the retrospective analysis of Sá et al. ${ }^{2}$

The tumor is encapsulated with slow and indolent development, being solitary in most cases. Initially, it is asymptomatic or causes local bulging or discomfort. ${ }^{4}$ Depending on the location, the evolution of the lesion may compress adjacent tissues generating paresthesia, Tinel's signal, pain, and dysesthesia. ${ }^{2}$ Motor deficit is reported in the literature as an indicator of malignancy; however, the patient described did not have such a characteristic. ${ }^{1}$

The manifestations of the peripheral nerve sheath tumors are nonspecific, requiring imaging for better tumor characterization and anatomopathological analysis to confirm the diagnosis, especially of the surgical resection product. ${ }^{3}$ Microscopically, schwannoma has areas of Antoni A (Verocay bodies) and Antoni B (mycelial matrix hypocellular), and abundant expression of S100 protein in histochemical analysis. ${ }^{2}$ Thus, differential diagnoses such as lipomas, neurofibromas, and gangliomas are discarded. ${ }^{2}$

Ultrasonographic (US) imaging is useful in tumor characterization, but it is more valuable intraoperatively when checking the presence of tumor residues. Besides that, like in our case, we used US imaging for evaluating the extension while studying the relation between the nerve and the lesion. Another justification is that nuclear magnetic resonance is the preferred modality for detailing mass dimensions and their differentiation in relation to adjacent tissues, aiding in the differentiation between benign and malignant tumor. ${ }^{2}$

The conduct of a suspected tumor mass of the peripheral nerve sheath may be expectant; however, this choice subjects the patient to a malignant transformation of the tumor, even though it is rare for the schwannomas. ${ }^{3,4}$ The symptoms, the progression of the lesion, and the discomforts make surgical resection the therapy of choice. ${ }^{1}$ This is not without risk, since the literature describes that in 26 to $51 \%$ of the cases there are new sensory disturbances and postoperative motors, mainly by manipulation or lesion of fascicles during the dissection. ${ }^{2,3}$
To avoid such injuries, a good definition of tumor extension in the preoperative period is necessary; in addition to the detailed knowledge of the anatomy, a wide incision that allows the visualization of healthy proximal and distal regions with circumferential dissection of the lesion to access to all surfaces and surrounding structures. ${ }^{3.4}$ And among the peripheral nerve sheath tumors, schwannomas are most frequently originated from a single neural bundle with reduced risk of injury compared with neurofibromas that involve multiple fascicles and malignant tumors.,

\section{Ethical Approval}

Ethical approval for the study was provided by Cajuru Hospital.

\section{Informed Consent}

An informed consent was obtained.

Funding

None.

Conflict of Interest

None declared.

\section{References}

1 Farid M, Demicco EG, Garcia R, et al. Malignant peripheral nerve sheath tumors. Oncologist 2014;19(2):193-201

2 Levi AD, Ross AL, Cuartas E, Qadir R, Temple HT. The surgical management of symptomatic peripheral nerve sheath tumors. Neurosurgery 2010;66(4):833-840

3 Ball JR, Biggs MT. Operative steps in management of benign nerve sheath tumors. Neurosurg Focus 2007;22(6):E7

4 Dusad T, Meena DS, Saini N, Sharma Y, Khurana D. Schwannoma of the Median Nerve at Mid Forearm Level. J Orthop Case Rep 2016;6(2):66-68

5 Hussain KMK, Beolchi G, Ayub NEC, Yvamot EY, Marques GN. Resection of retroperitoneal malignant peripheral nerve sheath tumor: case report. Arq Ciênc Saúde 2016;23(2):19-22 